\title{
Terapia breve e intensiva com finger kazoo em caso de disfonia orgânica pós-intubação orotraqueal
}

\author{
Short and intensive therapy with finger kazoo \\ in patient with organic dysphonia \\ post-orotracheal intubation
}

\section{Terapia breve e intensa con finger kazoo en paciente con disfonía orgánica postintubación orotraqueal}

\author{
Mara Keli Christmann* \\ Bruna Franciele da Trindade Gonçalves* \\ Joziane Padilha de Moraes Lima* \\ Gabriele Rodrigues Bastilha* \\ Fabricio Scapini* \\ Carla Aparecida Cielo*
}

\section{Resumo}

Objetivo: avaliar o efeito de um programa de Terapia Breve e Intensiva com a técnica finger kazoo em paciente adulta do sexo feminino com disfonia orgânica. Apresentação do caso clínico: realizadas medidas aerodinâmicas, acústicas, perceptivo auditivas, videolaringoestroboscópicas e autoavaliação vocal antes e após 15 sessões de terapia breve e intensiva com a técnica finger kazzo em uma mulher de 62 anos com disfonia orgânica por fibrose em prega vocal direita, fenda glótica do tipo irregular, com assimetria de fase e amplitude, além de edema da subglote e retração da porção anterior da prega vestibular direita decorrente de intubação orotraqueal. Resultados: Após a terapia, houve adequação dos tempos máximos de fonação, de medidas acústicas de frequência, perturbação de frequência, ruído,

* Universidade Federal de Santa Maria (UFSM), Santa Maria, Rio Grande do Sul, Brasil

Contribuição dos autores: MKC: coleta de dados, tabulação dos dados, elaboração do texto e correção do mesmo. BFTG: auxiliou na coleta dos dados e elaboração do texto. JPML: elaboração do texto, tabulação dos dados e interpretação da análise estatística. GRB: coleta de dados e elaboração do texto. FS: elaboração do texto e correção do mesmo. CAC: coordenadora da pesquisa, elaboração do texto, interpretação da análise estatística e correção do mesmo.

Email para correspondência: Mara Keli Christmann - marakchristmann@gmail.com Recebido: $17 / 06 / 2016$

Aprovado: 18/02/2017 
segmentos sub-harmônicos, melhor delineamento do traçado espectrográfico e de todos os parâmetros perceptivo auditivos, além de aumento dos escores de qualidade de vida, de depressão e ansiedade. Na avaliação videolaringoestroboscópica, houve melhora da amplitude de vibração da mucosa das pregas vocais e redução da constrição medial do vestíbulo laríngeo. Conclusão: houve modificações vocais positivas em nível glótico, respiratório e ressonantal, bem como no exame videolaringoestroboscópico e na autoavaliação vocal, evidenciando que essa foi uma modalidade de tratamento efetiva para o caso.

Palavras-chave: Voz; Fonoterapia; Disfonia

\section{Abstract}

Objective: to evaluate the effect of a program of brief and intensive therapy with finger kazoo technique in adult female patient with organic dysphonia. Presentation of the case: were realized aerodynamic, acoustic, perceptual, videolaryngostroboscopic measures and vocal self-assessment before and after 15 sessions of short and intensive therapy with finger kazzo technique in a 62 years woman with organic dysphonia by fibrosis in the right vocal fold, glottis of irregular type with phase asymmetry and amplitude, and edema of the glottis and contraction of the anterior portion of the right vestibular fold due to endotracheal intubation. Results: After therapy, there was improvement of maximum phonation time, acoustic frequency measurements, frequency of disturbance noise, sub-harmonic segments, improved stroke spectrographic and all perceptual parameters, as well as improvement of quality of life scores, depression and anxiety. In the videolaryngostroboscopy evaluation, there was improvement in range of mucosa vibration of the vocal folds and reduction of medial constriction of the laryngeal vestibule. Conclusion: there were positive vocal changes in glottic, respiratory and resonant level, videolaryngostroboscopy and vocal self-assessment, emphasizing that this was an effective treatment modality for the case.

Keywords: Voice; Speech therapy; Dysphonia

\section{Resumen}

Objetivo: evaluar los efectos de un programa de Terapia Breve e Intensa utilizando la técnica finger kazoo en una paciente adulta del sexo femenino con disfonía orgánica. Presentación del caso clínico: fueron realizadas las medidas aerodinámicas, acústicas, perceptivo auditivas, videolaringoestroboscópicas y autoevaluación vocal antes y post 15 sesiones de terapia breve e intensa con la técnica finger kazzo en una mujer de 62 años con disfonía orgánica por fibrosis en la cuerda vocal derecha, hiato glótico irregular, con asimetría de fase y amplitud, además de edema de la subglotis y retracción de la porción anterior de la cuerda vestibular derecha debido a intubación orotraqueal. Resultados: Después de la terapia, hubo adecuación en los tiempos máximos de fonación, de las medidas acústicas de frecuencia, perturbación de frecuencia, ruido, segmentos sub-armónicos, mejora del trazado espectrográfico y de todos los parámetros perceptivo auditivos, así como mejora de los puntajes de calidad de vida, depresión y ansiedad. En la evaluación videolaringoestroboscópica, hubo mejora en la amplitud de vibración da la mucosa de las cuerdas vocales y reducción da la constricción mediana del vestíbulo laríngeo. Conclusión: hubo positivos cambios vocales a nivel glótico, respiratorio y resonante, así como en el examen videolaringoestroboscópico y en la autoevaluación vocal, lo que enseña que la modalidad de tratamiento fue efectiva para el caso.

Palabras clave: Voz; Logoterapia; Disfonía 
A intubação endotraqueal permite a assistência ventilatória em pacientes anestesiados ou sob ventilação mecânica, entretanto, em alguns casos gera comprometimentos na região posterior da laringe, nos processos vocais das cartilagens aritenoides, região em que fica posicionado o tubo oro ou nasotraqueal, podendo resultar em ulceração que envolve o epitélio da mucosa interaritenoidea, cicatrização com fibrose e fixação das cartilagens aritenoides na linha mediana, podendo afetar a voz ${ }^{1,2}$.

Existem três fatores de risco para as complicações pós-intubações endotraqueais que são: fatores relacionados ao paciente, tais como idade avançada, pois a mucosa laríngea se torna mais frágil, história de intubação e tabagismo; fatores associados às condições técnicas para atingir e manter a intubação, tais como duração prolongada, o tamanho do tubo endotraqueal, agitação do paciente, mau posicionamento da sonda (colocada muito acima ou muito abaixo da glote, com um balão localizado no anel cricoideo), pobre umidificação do ar inspirado e a infecção local; e os fatores relacionados ao médico, como a falta de experiência e a dificuldade de colocação do tubo endotraqueal no lugar apropriado ${ }^{1,2}$.

Grande parte das lesões laríngeas pós-intubação se resolve espontaneamente pela capacidade de regeneração do epitélio. No entanto, em determinadas circunstâncias, que levam à diminuição da perfusão tecidual e cicatrização deficiente, o processo pode originar lesões laríngeas de gravidade variável ${ }^{2}$.

A cicatrização das lacerações de mucosa da laringe pode ocorrer à custa de aderências e fibroses. Quando se instalam na comissura anterior da glote, promovem grande prejuízo à voz por comprometer a porção fonatória ${ }^{1,2}$. Nesses casos, necessita-se realizar fonoterapia que visa a atingir a melhor voz possível.

A literatura destaca, atualmente, a realização de terapia breve e intensiva (TBI), uma modalidade de tratamento que pode ser utilizada em casos de disfonia e visa à restauração eficiente e eficaz da melhor voz possível em um curto período de tempo ${ }^{3}$. As técnicas vocais, como o Finger Kazoo (FK), proporcionam melhoras na voz, como tem sido mostrado por alguns estudos ${ }^{4-7}$. Assim, a TBI com a prática diária da(s) técnica(s) eleita(s) para a fonoterapia favorece a potencialização dos efeitos benéficos da técnica, mantendo esse novo ajuste em longo $\operatorname{prazo}^{3,8,9}$.

Em relação ao $\mathrm{FK}$, técnica escolhida para o presente estudo de caso, durante sua execução ocorre aumento da pressão aérea supraglótica, o que gera ressonância retroflexa e eleva a pressão ao nível da glote, levando ao afastamento das pregas vocais e reduzindo o impacto quando elas entram em contato medialmente ${ }^{5,7,10}$. Estudos científicos mostram, ainda, que essa técnica proporciona adequada autopercepção vocal ${ }^{5,7,10}$, da ressonância vocal e do tipo de voz, com maior estabilidade da emissão. Análises acústicas evidenciam diminuição do ruído e maior energia harmônica pelo provável aumento da qualidade de vibração da onda mucosa das pregas vocais 5,7 .

Assim, o objetivo deste trabalho foi avaliar o efeito de um programa de TBI com a técnica FK em uma paciente adulta do sexo feminino com disfonia orgânica por fibrose em prega vocal direita, fenda glótica do tipo irregular, com assimetria de fase e amplitude, além de edema da subglote e retração da porção anterior da prega vestibular direita decorrente de intubação orotraqueal.

\section{Método}

Este trabalho caracteriza-se por ser um estudo de caso, que teve aprovação prévia do Comitê de Ética em Pesquisa da Universidade Federal de Santa Maria ( $\left.n^{\circ} 016945 / 2010-76\right)$ e a paciente em questão foi convidada a ler e assinar o Termo de Consentimento Livre e Esclarecido (TCLE), como recomenda a resolução 466 da Comissão Nacional de Ética em Pesquisa - CONEP/2012.

A paciente C., sexo feminino, cirurgiã-dentista aposentada, 62 anos de idade, $56 \mathrm{Kg}, 1,67 \mathrm{~m}$, compareceu a um Serviço de Fonoaudiologia com queixa de rouquidão, fadiga e falhas na voz. A mesma negou tabagismo e ingestão de álcool. Com relação à saúde geral, havia realizado tratamento prévio endocrinológico e intervenção cirúrgica com relato de intubação orotraqueal, devido a nódulo na glândula tireoide e tratamento otorrinolaringológico para sinusite. Na ocasião, realizava tratamento psiquiátrico, devido a Transtorno Bipolar. Além disso, não apresentava particularidades com relação à avaliação do sistema estomatognático e à triagem auditiva (audiômetro Fonix FA 12 Digital, Frye Electronics, Estados Unidos). 


\section{Avaliações realizadas}

Todas as avaliações mencionadas a seguir foram realizadas antes e após a TBI com FK para a comparação dos resultados.

Autoavaliação vocal: a paciente preencheu os protocolos Perfil de Participação e Atividade Vocais (PPAV) 11,12,13 11-13; Qualidade de Vida em Voz (QVV) ${ }^{13}$; Índice de Desvantagem Vocal (IDV) 13; Escala de Sintomas Vocais (ESV) ${ }^{13,14}$ e a Escala Hospitalar de Ansiedade e Depressão (EHAD) ${ }^{15}$. Além disso, preencheu outro protocolo (Anexo 1) com diversas opções de sensações vocais positivas e negativas, distribuídas aleatoriamente, em que poderia marcar quantas estivesse sentindo ${ }^{4,10}$. Utilizaram-se como pontos de corte os seguintes valores: no PPAV, para o escore total em casos de não professores como a paciente em questão, o ponto de corte foi de $4,5^{12}$; no QVV, aspecto socioemocional 90,65 , físico 89,6 e total 91,25 13; no IDV, aspecto emocional 3, funcional 7,5, orgânico 10,5 e total $19^{13}$; na ESV, aspecto físico 6,5 , limitação 11,5 , emocional 1,5 e total $16^{13}$; na EHAD, consideraram-se 11 pontos como valor de corte tanto para ansiedade como para depressão ${ }^{16}$.

Medidas aerodinâmicas: foram coletados os tempos máximos de fonação (TMF) dos fonemas /a/, /i/, /u/, /e/, /s/ e /z/ ${ }^{17}$, após uma inspiração profunda, em pitch, loudness e qualidade vocal habituais, em posição ortostática e em uma só expiração, além do /e/ áfono (/è/), nas mesmas condições. Cada emissão foi realizada por duas vezes e considerou-se o maior valor cronometrado em segundos ${ }^{18,19}$.

Todas as emissões foram gravadas com microfone profissional omnidirecional ECM 8000 (Behringer, Alemanha) (resposta de frequência plana de $15 \mathrm{~Hz}$ a $20 \mathrm{kHz}$ ), acoplado ao gravador digital profissional H4n (Zoom, Estados Unidos) (96 kHz, 16 bits, e configurado em 50\% do nível de captação do sinal de entrada), que foi posicionado a uma distância de quatro centímetros em frente à boca para as vogais sustentadas e de dez centímetros para os fricativos, com ângulo de $90^{\circ}$ em relação à boca ${ }^{17-19}$.

Coletou-se a capacidade vital forçada (CVF) com espirômetro digital portátil Spirobank II ( $M e$ dical International Research, Itália). A paciente permaneceu sentada, o bocal, inserido na turbina em $0,5 \mathrm{~cm}$, foi colocado na boca da paciente e o clip nasal foi colocado no nariz. Após, C. realizou uma inspiração máxima seguida de uma expiração máxima no aparelho. Considerou-se o maior valor obtido dentre três coletas realizadas com oclusão nasal.

A partir do maior valor da CVF, foi calculada a relação entre o TMF obtido (TMFO) (maior valor do TMF/a/) e o TMF previsto (TMFP) que resultou da multiplicação do valor da CVF pelo valor $0,0051^{18}$. Após, o TMFO foi dividido pelo TMFP, considerando-se como normalidade os valores entre 0,9 e 1,1 . Valores inferiores foram considerados sugestivos de escape aéreo à fonação e valores superiores sugestivos de hiperfunção glótica ${ }^{18}$. O cálculo do Coeficiente Fônico Simples (CFS) foi realizado dividindo-se o valor da maior CVF pelo maior TMF/a/.

Durante a emissão da vogal /a/, foi aferido o nível de pressão sonora (NPS) (aparelho Icel, DL-4200, Brasil), posicionado a $30 \mathrm{~cm}$ em frente à boca e regulado no circuito de ponderação - "A" e circuito de resposta - "lenta $-S L O W{ }^{18}$.

Medidas vocais acústicas: o $\mathrm{TMF} / \mathrm{a} /$ foi editado, retirando-se o ataque vocal e o final da emissão, resultando em uma janela de tempo de $6 \mathrm{~s}$, com taxa de amostragem de $44 \mathrm{kHz}$ e $16 \mathrm{bits}$, analisada através do Multi Dimensional Voice Program Advanced (MDVPA) (Kay Pentax ${ }^{\circledR}$, Estados Unidos). Foram extraídas as medidas de frequência: frequência fundamental (f0), f0 máxima (fhi), f0 mínima (flo), Desvio-padrão da f0 (STD); as medidas de perturbação de frequência: Jitter absoluto (Jita), Jitter percentual ou relativo (Jitt), Média relativa da perturbação do pitch (RAP), Quociente de perturbação do pitch ou da frequência (PPQ), Quociente de perturbação do pitch ou da frequência suavizado (sPPQ), Variação da f0 (vf0); as medidas de perturbação de amplitude: Shimmer absoluto ou em dB (ShdB), Shimmer percentual ou relativo (Shim), Quociente de perturbação da amplitude (APQ), Quociente de perturbação da amplitude suavizado (sAPQ), Variação da amplitude (vAm); as medidas de ruído: Proporção ruído-harmônico (NHR), Índice de turbulência vocal (VTI), Índice de fonação suave (SPI); as medidas de quebra de voz: Grau de quebras vocais (DVB), Número de quebras vocais (NVB); as medidas de segmentos surdos ou não sonorizados: Número de segmentos não sonorizados (NUV), Grau de segmentos não sonorizados (DUV); as medidas de segmentos sub-harmônicos: Grau dos componentes sub-harmônicos (DSH), Número de segmentos sub-harmônicos 
(NSH). Para a medida de f0, foram utilizados os valores de referência para o sexo feminino de 150 a $250 \mathrm{~Hz}^{19}$.

Ainda, analisou-se o TMF/a/ editado no programa Real Time Spectrogram (RTS) (Kay Pentax ${ }^{\circledR}$, Estados Unidos), através do qual foram extraídos os traçados espectrográficos de banda larga (EBL), filtrados em 100 points $(646,00 \mathrm{~Hz})$ e EBE com filtro de 1024 points $(63,09 \mathrm{~Hz})$, com taxa de amostragem de $11 \mathrm{kHz}, 16$ bits e resolução de $5 \mathrm{kHz}$.

Os parâmetros considerados na EBL foram: escurecimento do traçado do primeiro formante (F1), F2, F3 e F4, escurecimento do traçado das baixas, médias e altas frequências; definição do F1, F2, F3 e F4; regularidade do traçado; e presença de ruído das baixas, médias e altas frequências. Os parâmetros considerados na EBE foram: escurecimento do traçado das baixas, médias e altas frequências; substituição de harmônicos por ruído nas baixas, médias e altas frequências; presença de ruído entre os harmônicos nas baixas, médias e altas frequências; definição de harmônicos das baixas, médias e altas frequências; regularidade do traçado das baixas, médias e altas frequências; número de harmônicos das baixas, médias e altas frequências e presença de sub-harmônicos $5,7,20$.

As espectrografias foram analisadas visual e individualmente por três fonoaudiólogos juízes com experiência na área de voz e análise acústica, com base em protocolo específico. Cada aspecto descrito anteriormente foi avaliado utilizando-se uma escala analógicovisual que apresentava $10 \mathrm{~cm}$ de extensão (de zero a $100 \mathrm{~mm}$ ) e os juízes realizaram a marcação do grau do desvio considerando a extremidade esquerda (zero) como ausência total do parâmetro avaliado e a direita como o grau máximo do parâmetro em questão, assim o número de milímetros marcado na escala pôde ser convertido em porcentagem. Os juízes receberam, juntamente com as imagens espectrográficas para análise, uma espectrografia-âncora, considerada normal, para reduzir a subjetividade da avaliação ${ }^{5,7,20}$. Para fins de resultado, considerou-se a média da avaliação dos três juízes.

Avaliação de videolaringoestroboscopia: realizada por médico otorrinolaringologista (ORL), utilizando aparelho Atmos (Lenzkirch, Alemanha) com óptica Storz de $70^{\circ}$ (Tuttlingen, Alemanha). Durante o exame, C. permaneceu sentada, com a cabeça levemente inclinada para frente e para cima e emitiu, de forma sustentada, as vogais /e/ e /i/, além de duas emissões da fonação reversa. $\mathrm{O}$ diagnóstico Otorrinolaringológico foi: "Fibrose na prega vocal direita, fenda glótica do tipo irregular, com assimetria de fase e amplitude, além de edema da subglote e retração da porção anterior da prega vestibular direita".

Após o término da terapia, na reavaliação, a paciente foi encaminhada pelo médico ORL para avaliação com médico neurologista, a fim de realizar uma eletroneuromiografia laríngea, devido a suspeita de paralisia do nervo laríngeo superior bilateral secundária à cirurgia de tireoide.

As imagens pré e pós-fonoterapia foram editadas e, posteriormente, avaliadas em consenso e simultaneamente por três ORL juízes, com experiência em laringologia de no mínimo cinco anos, utilizando uma escala analógicovisual de $10 \mathrm{~cm}$ (o número de milímetros marcado na escala foi convertido em porcentagem) para os aspectos: amplitude de vibração da mucosa, constrição do vestíbulo laríngeo (medial, ântero-posterior, global), simetria da vibração e presença de onda mucosa ${ }^{21}$.

Avaliação vocal perceptivo auditiva: realizada com base na emissão do TMF/a/, através da escala de avaliação vocal perceptivo auditiva RASATI ${ }^{22}$, que avalia rouquidão, aspereza, soprosidade, astenia, tensão e instabilidade. Essa avaliação foi realizada individualmente e separadamente por três fonoaudiólogos juízes com experiência de no mínimo cinco anos na área de voz. A escala utilizada foi proposta originalmente para ser avaliada com a gradação de "0", "1', "2" e "3", entretanto como a literatura atual tem apontado um grande avanço nas avaliações utilizando-se de escalas analógico-visuais ${ }^{7,21}$, optou-se por realizar uma adaptação no protocolo original. Desta forma, foram utilizados os parâmetros avaliados pela escala RASATI, porém com a gradação de uma escala analógicovisual de $10 \mathrm{~cm}$, onde zero equivale à ausência do parâmetro e 10 à alteração extrema (o número de milímetros marcado na escala foi convertido em porcentagem). Para fins de resultado, considerou-se a média da avaliação dos três juízes.

Ressalta-se que todos os juízes da pesquisa foram cegados a respeito do objetivo e momento das diferentes avaliações (pré e pós-fonoterapia), tendo conhecimento somente da idade e sexo da paciente ${ }^{4}$. 
Terapia

A avaliação pré-terapia foi realizada numa sexta-feira, após uma semana de uso da voz no ambiente profissional, sendo a fonoterapia iniciada no mesmo dia, com duração de 15 dias, havendo um intervalo de dois dias (sábado e domingo) por semana ${ }^{3,23}$. A avaliação pós-terapia foi realizada após a décima quinta sessão de terapia que ocorreu numa quinta-feira ${ }^{23}$.

As sessões foram realizadas por terapeutas diferentes (fonoaudiólogos ou acadêmicos do curso de Fonoaudiologia) previamente treinados ${ }^{3,15}$ que se revezaram nos atendimentos ${ }^{3}$.

Cada sessão de terapia teve a seguinte estrutura: execução da técnica FK, realizando um sopro sonorizado, com os lábios arredondados (semelhante à emissão da vogal /u/) em loudness e pitch habituais, evitando inflar as bochechas ou hipertensionar a musculatura lingual. Ao mesmo tempo, o dedo indicador deveria estar posicionado verticalmente sobre os lábios, tocando-os levemente, mas sem pressão. Deveria ocorrer um ruído secundário de fricção pelo contato do fluxo de ar com o dedo indicador ${ }^{4,5,10}$.

Cada emissão da técnica foi realizada em TMF e C. realizou seis séries de 15 repetições da técnica de $\mathrm{FK}^{24}$. Ao final de cada série, a paciente teve um intervalo de um minuto de repouso passivo (silêncio absoluto) ${ }^{24}$. Ao longo da sessão, a água ficou disponível para ser ingerida livremente em função do grande fluxo aéreo envolvido na execução da técnica $\mathrm{FK}^{4,5}$.

Ao longo da realização da técnica, C. permaneceu sentada, com os pés apoiados no chão, coluna ereta, sem deslocamento cervical, com ângulo de $90^{\circ}$ entre o queixo e o pescoço, sem aumento da contração muscular de cintura escapular e região suprahioidea, mantendo o ritmo constante entre uma repetição e outra, sem fazer uso da reserva expiratória. Ainda, controlou-se a flutuação ou variabilidade de pitch e/ou loudness, que deveria ser evitada. Uma terapeuta permaneceu monitorando esses aspectos ao longo de cada sessão ${ }^{4,18,19}$.

C. foi instruída a realizar a técnica somente nas sessões, sem treino em casa, a fim de evitar sua execução sem o monitoramento do terapeuta ${ }^{3}$.

\section{Resultados}

A tabela 1 evidencia os valores das medidas aerodinâmicas, nos momentos pré e pós-terapia.

A tabela 2 apresenta os resultados da análise vocal acústica por meio do programa MDVPA nos momentos pré e pós-terapia.

Na tabela 3, podem ser visualizados os valores dos protocolos de qualidade de vida e voz nos momentos pré e pós-terapia.

A tabela 4 evidencia os parâmetros vocais acústicos espectrográficos de banda larga e estreita nos momentos pré e pós-terapia.

$\mathrm{Na}$ tabela 5, podem ser visualizados os resultados da avaliação vocal perceptivo auditiva nos momentos pré e pós-terapia.

A tabela 6 mostra os resultados da avaliação videolaringoestroboscópica nos momentos pré e pós-terapia. 
Tabela 1. Resultados descritivos das medidas aerodinâmicas

\begin{tabular}{cccc}
\hline Medidas & Pré-terapia & Pós-terapia & Esperado \\
\hline TMF /a/(s) & 12,5 & 16,64 & 15 a 25 \\
TMF /i/(s) & 11,42 & 17,74 & 15 a 25 \\
TMF /u/(s) & 11,8 & 16,74 & 15 a 25 \\
TMF /s/(s) & 7,43 & 14,87 & 15 a 25 \\
TMF /z/(s) & 8,2 & 14,56 & 15 a 25 \\
TMF /e/(s) & 11,4 & 16,44 & 15 a 25 \\
TMF /è/(s) & 7,95 & 21,34 & 15 a 25 \\
Relação s/z & 0,9 & 1,1 & 0,8 a 1,2 \\
Relação è/e & 0,69 & 1,29 & 0,8 a 1,2 \\
CV(ml) & 3650 & 3650 & 2600 \\
NPS modal (dBA) & 69 & 62,4 & - \\
TMFO(s) & 12,5 & 16,64 & 18,61 \\
TMFP(s) & 18,61 & 18,61 & 18,61 \\
TMFO/TMFP & 0,76 & 0,9 & 0,9 a 1,1 \\
CFS(ml/s) & 292 & 219,35 & 105 a 256 \\
\hline
\end{tabular}

Legenda: TMF: tempo máximo de fonação; CV: capacidade vital; NPS: nível de pressão sonora; TMFO: tempo máximo de fonação obtido; TMFP: tempo máximo de fonação previsto; CFS: coeficiente fônico simples; -: não há valor de normalidade.

Tabela 2. Análise descritiva dos protocolos de qualidade de vida e voz

\begin{tabular}{|c|c|c|c|c|}
\hline Protocolo & Pré-terapia & Pós-terapia & $\begin{array}{l}\text { Pontuação } \\
\text { máxima }\end{array}$ & $\begin{array}{c}\text { Ponto de } \\
\text { corte }\end{array}$ \\
\hline EHAD ansiedade & 12,00 & 10,00 & 21 & 11 \\
\hline EHAD depressão & 8,00 & 4,00 & 21 & 11 \\
\hline QVV socioemocional & 43,75 & 56,25 & 100 & 90,65 \\
\hline QVV físico & 45,8 & 50 & 100 & 89,6 \\
\hline QVV total & 45 & 57,5 & 100 & 91.25 \\
\hline IDV total & 59,00 & 56,00 & 120 & 19 \\
\hline IDV funcional & 21,00 & 20,00 & 40 & 7,5 \\
\hline IDV orgânico & 20,00 & 25,00 & 40 & 10,5 \\
\hline IDV emocional & 18,00 & 11,00 & 40 & 3 \\
\hline PPAV total & 200,00 & 115,00 & 280 & 4,5 \\
\hline PPAV autopercepção do problema de voz & 8,00 & 9,00 & 10 & - \\
\hline PPAV efeitos trabalho & 10,00 & 2,00 & 40 & - \\
\hline PPAV efeitos comunicação diária & 84,00 & 57,00 & 120 & - \\
\hline PPAV efeitos comunicação social & 31,00 & 6,00 & 40 & - \\
\hline PPAV efeitos emoção & 67,00 & 41,00 & 70 & - \\
\hline ESV total & 90,00 & 76,00 & 120 & 16 \\
\hline ESV limitação & 39,00 & 47,00 & 60 & 11,5 \\
\hline ESV emocional & 23,00 & 8,00 & 32 & 1,5 \\
\hline ESV físico & 20,00 & 18,00 & 28 & 6,5 \\
\hline Sensações positivas & 0 & 2 & - & - \\
\hline Sensações negativas & 12 & 12 & - & - \\
\hline
\end{tabular}

Legenda: EHAD: Escala Hospitalar de Ansiedade e Depressão; QVV: Qualidade de Vida em Voz; IDV: Índice de Desvantagem Vocal; PPAV: Perfil de Participação e Atividade Vocais; ESV: Escala de Sintomas Vocais; -: não há ponto de corte ou pontuação máxima. 
Tabela 3. Análise descritiva das medidas acústicas com o Multi Dimensional Voice Program Advanced (Kay Pentax $®$ )

\begin{tabular}{cccc}
\hline Parâmetro & Pré-terapia & Pós-terapia & Normalidade \\
\hline fO (Hz) & 118,82 & 75,6 & 243,973 \\
fhi (Hz) & 150,43 & 78,13 & 252,724 \\
flo (Hz) & 69,41 & 72,47 & 234,861 \\
STD (Hz) & 42,08 & 1 & 2,722 \\
Jita (ms) & 166,05 & 107,35 & 26,927 \\
Jitt (\%) & 1,76 & 0,81 & 0,633 \\
RAP (\%) & 1,08 & 0,45 & 0,378 \\
PPQ (\%) & 1,26 & 0,53 & 0,366 \\
SPPQ (\%) & 7,27 & 0,99 & 0,532 \\
vfO (\%) & 35,41 & 1,32 & 1,149 \\
ShdB (dB) & 0,77 & 0,9 & 0,176 \\
Shim (\%) & 8,99 & 10,44 & 1,997 \\
APQ (\%) & 6,83 & 8,95 & 1,397 \\
SAPQ (\%) & 9,54 & 13,56 & 2,371 \\
vAm (\%) & 18,46 & 24,95 & 10,743 \\
NHR & 0,16 & 0,18 & 0,112 \\
VTI & 0,02 & 0,06 & 0,046 \\
SPI & 13,31 & 7,8 & 7,534 \\
DVB (\%) & 0 & 0 & 0,200 \\
NVB (\%) & 0 & 0 & 0,200 \\
DUV (\%) & 7,76 & 10,34 & 0,200 \\
NUV & 9 & 12 & 0,200 \\
DSH (\%) & 4,67 & 0 & 0,200 \\
NSH & 5 & 0 & 0,200 \\
\hline
\end{tabular}

Legenda: f0: frequência fundamental; fhi: frequência fundamental máxima; flo: frequência fundamental mínima; STD: Desvio-padrão da frequência fundamental; Jitta: jitter absoluto; Jitt: jitter percentual ou relativo; RAP: Média relativa da perturbação do pitch; PPQ: Quociente de perturbação do pitch ou da frequência; SPPQ: Quociente de perturbação do pitch ou da frequência suavizado; vf0:

Variação da frequência fundamental; ShdB: Shimmer absoluto; Shim: Shimmer percentual ou relativo; APQ: Quociente de perturbação da amplitude; sAPQ: Quociente de perturbação da amplitude suavizado; vAm: Variação da amplitude; NRH: Proporção ruído-

harmônico; VTI: Índice de turbulência vocal; SPI: Índice de fonação suave; DVB: Grau de quebras vocais; NVB: Número de quebras vocais; DUV: Número de segmentos não sonorizados; NUV: Grau de segmentos não sonorizados; DSH: Grau dos componentes subharmônicos; NSH: Número de segmentos sub-harmônicos 
Tabela 4. Análise descritiva dos dados vocais acústicos espectrográficos obtidos pelo programa Real Time Spectrogram (Kay Pentax $®$ )

\begin{tabular}{|c|c|c|c|c|c|}
\hline Banda larga $(\%)$ & $\begin{array}{c}\text { Pré- } \\
\text { terapia }\end{array}$ & $\begin{array}{c}\text { Pós- } \\
\text { terapia }\end{array}$ & Banda estreita (\%) & $\begin{array}{c}\text { Pré- } \\
\text { terapia }\end{array}$ & $\begin{array}{c}\text { Pós- } \\
\text { terapia }\end{array}$ \\
\hline Escurecimento traçado F1 & 50 & 60 & $\begin{array}{l}\text { Escurecimento traçado } \\
\text { baixas e médias } \mathrm{f}\end{array}$ & 60 & 60 \\
\hline Escurecimento traçado F2 & 60 & 50 & Escurecimento altas $f$ & 20 & 20 \\
\hline Escurecimento traçado F3 & 30 & 30 & Ruído baixas e médias $f$ & 60 & 60 \\
\hline Escurecimento traçado F4 & 20 & 20 & Ruído altas $\mathrm{f}$ & 30 & 20 \\
\hline Escurecimento traçado altas $f$ & 20 & 20 & $\begin{array}{l}\text { Substituição de h por ruído } \\
\text { nas baixas e médias f }\end{array}$ & 50 & 50 \\
\hline $\begin{array}{l}\text { Escurecimento traçado das } \\
\text { baixas e médias f }\end{array}$ & 50 & 50 & $\begin{array}{l}\text { Substituição de h por ruído } \\
\text { altas } \mathrm{f}\end{array}$ & 30 & 20 \\
\hline Ruído nas baixas e médias $f$ & 70 & 60 & $\begin{array}{l}\text { Definição de } \mathrm{h} \text { nas baixas e } \\
\text { médias } \mathrm{f}\end{array}$ & 40 & 30 \\
\hline Ruído nas altas $f$ & 50 & 40 & Definição de h nas altas $f$ & 20 & 10 \\
\hline Definição de F1 & 30 & 50 & $\begin{array}{l}\text { Regularidade traçado nas } \\
\text { baixas e médias } f\end{array}$ & 40 & 40 \\
\hline Definição de F2 & 40 & 40 & Regularidade traçado altas $f$ & 20 & 10 \\
\hline Definição de F3 & 20 & 3 & $\begin{array}{l}\text { Número } \mathrm{h} \text { nas baixas e } \\
\text { médias } \mathrm{f}\end{array}$ & 50 & 50 \\
\hline Definição F4 & 10 & 20 & Número de $h$ nas altas $f$ & 20 & 20 \\
\hline Regularidade do traçado & 60 & 50 & Presença de sub-h & 0 & 0 \\
\hline
\end{tabular}

Legenda: F: formante; f: frequência; h: harmônico

Tabela 5. Análise descritiva das características vocais perceptivo auditivas pela escala RASATI

\begin{tabular}{ccc}
\hline Parâmetro (\%) & Pré-terapia & Pós-terapia \\
\hline Rouquidão & 53,33 & 30 \\
Aspereza & 28,33 & 21,66 \\
Soprosidade & 26,66 & 20 \\
Astenia & 3,33 & 3,33 \\
Tensão & 30 & 0 \\
Instabilidade & 15 & 5 \\
\hline
\end{tabular}

Tabela 6. Análise descritiva da avaliação videolaringoestroboscópica

\begin{tabular}{ccc}
\hline Parâmetro (\%) & Pré-terapia & Pós-terapia \\
\hline $\begin{array}{c}\text { Amplitude de } \\
\text { Vibração da mucosa } \\
\text { Constrição medial } \\
\text { do vestíbulo } \\
\text { laríngeo }\end{array}$ & 60 & 80 \\
$\begin{array}{c}\text { Constrição ântero- } \\
\text { posterior do } \\
\text { vestíbulo laríngeo } \\
\begin{array}{c}\text { Constrição global } \\
\text { do vestíbulo } \\
\text { laríngeo }\end{array}\end{array}$ Simetria da $_{\text {vibração }}$ & 0 & 0 \\
$\begin{array}{c}\text { Presença de onda } \\
\text { mucosa }\end{array}$ & 0 & 0 \\
\hline
\end{tabular}




\section{Discussão}

Após a TBI com FK, houve aumento dos TMF das vogais, fricativos e do /è/, (Tabela 1) que pode estar relacionado tanto à melhora do nível respiratório, quanto fonatório, e sua integração.

Durante a execução do FK, faz-se necessário manter o fluxo aéreo, sem aumentar a tensão ao nível das pregas vocais; com isso, é possível que o FK influencie a musculatura respiratória, com melhora no controle de sua descontração progressiva durante a emissão. Isso foi evidenciado pelo aumento do valor dos TMF, salientando-se o aumento do /è e do fricativo não-vozeado /s/ que não se utilizam de fonte glótica (Tabela 1) ${ }^{25}$.

$\mathrm{O}$ aumento dos demais TMF demonstra maior coordenação pneumofônica adquirida pela paciente, possivelmente devido à melhora do suporte aéreo e às modificações geradas pela técnica, de acordo com a literatura, como aumento do movimento muco-ondulatório, melhora da sincronia da vibração das pregas vocais e aumento do contato da mucosa com menos esforço ${ }^{4,10}$, o que é corroborado pelos resultados obtidos na análise acústica em relação à diminuição de todas as medidas de jitter $\mathrm{e}$ das medidas NHR, SPI, DSH e NSH, relacionadas à presença de ruído à emissão (Tabela 3 ).

Pesquisa com o Programa de Exercícios Funcionais Vocais de Stemple e Gerdeman foi realizada com 17 professoras, tendo duração de oito semanas, com um encontro semanal. Verificou-se que, todas as participantes apresentaram aumento do TMF, de maneira que seis apresentaram valores dentro dos padrões de normalidade no momento pós-terapia e as demais apresentaram valores entre dez e $14 \mathrm{~s}$ ${ }^{26}$. Nesse estudo, todos os TMF estavam abaixo da normalidade esperada para o sexo feminino de 15 a $25 \mathrm{~s}{ }^{18} \mathrm{e}$, após a fonoterapia, todos aumentaram acima de $14 \mathrm{~s}$ (Tabela1).

Indo ao encontro dos presentes resultados, um artigo sobre TBI (nove sessões em três semanas), com dez mulheres que apresentavam nódulos vocais mostrou aumento do TMF/a/ ${ }^{8}$. Ressalta-se, porém, que a investigação citada utilizou também exercícios respiratórios durante a terapia. No presente estudo não foram utilizados exercícios respiratórios, de forma que as melhoras sobre o nível respiratório se devem apenas aos efeitos do FK.

O CFS diminuiu, e a relação TMFO/TMFP (Tabela 1) aumentou, apresentando valor dentro do limite de normalidade, entre 0,9 e 1,1. A literatura refere que a interpretação conjunta dos dados aerodinâmicos e tais resultados inferem diminuição de escape aéreo à fonação na reavaliação. Assim, é possível que a paciente tenha melhorado sua coordenação pneumofonoarticulatória com a terapia proposta ${ }^{27}$.

No MDVPA, observou-se melhora das medidas de frequência, como redução do STD da f0, aumento de flo e redução de fhi, o que evidencia redução da variabilidade da f0, diminuição de todas as medidas de Jitter, do SPI, NSH e DSH (Tabela 2). Isso pode ter ocorrido devido ao aumento da periodicidade e estabilidade do sinal vocal e do controle neuromuscular ocasionado pelo FK. Tais resultados são semelhantes aos de recente pesquisa que verificou o efeito de uma sessão de terapia utilizando FK em professoras com e sem afecção laríngea (AL) e mostrou redução de medidas de frequência (fhi), Jitter (sPPQ), Shimmer (Shim e APQ) e quebras vocais (NVB) no grupo que não apresentava $\mathrm{AL}$, após uma sessão com FK ${ }^{7}$. No grupo com $\mathrm{AL}$, uma sessão de terapia não foi suficiente para gerar modificações significativas na análise acústica ${ }^{7}$.

Em outra investigação, a técnica $\mathrm{FK}$ foi realizada em três séries de 15 repetições, por 46 mulheres sem AL e sem queixas vocais. Após a execução, houve diminuição do ruído, aumento da f0, da estabilidade vocal e da energia harmônica ${ }^{4}$.

Outros estudos, que realizaram uma modalidade de TBI em pacientes com nódulos vocais, embora usando outras técnicas vocais, também evidenciaram resultados positivos como a redução significativa de medidas de jitter e shimmer e aumento da f0 ${ }^{8,9}$, além de redução da NHR ${ }^{8}$.

Em relação à análise vocal acústica espectrográfica, verificou-se, na EBL, melhora no escurecimento do traçado de F1, no ruído das baixas, médias e altas frequências e na definição de F1 e F4. Na EBE, houve melhora do ruído nas altas frequências e da substituição de harmônico por ruído nas altas frequências (Tabela 4). Esses resultados evidenciam parcialmente a diminuição da energia aperiódica e aumento da energia harmônica, com melhora da ressonância vocal da paciente, apesar de também ter sido evidenciada diminuição da definição dos harmônicos nas baixas, médias e altas frequências (EBE) e de F3 (EBL), diminuição da regularidade do traçado nas altas frequências (EBE) e de todo o traçado (EBL) e diminuição do escurecimento de F2 (EBL). Tais 
aspectos concordam parcialmente com pesquisa que verificou o efeito imediato do FK em mulheres sem disfonia ${ }^{5}$. No estudo citado, houve melhora da intensidade do escurecimento do traçado dos formantes (F) e das altas frequências, regularidade do traçado e definição dos harmônicos, além de melhora na autoavaliação vocal, conforme aumento da intensidade do escurecimento do traçado de F3, da definição do traçado, do número de harmônicos e redução da substituição de harmônicos por ruído nas médias frequências ${ }^{5}$.

Antes da terapia, a paciente não apresentava qualquer sensação positiva em relação à sua voz e, após, referiu duas sensações positivas (voz solta para falar e sensação de projeção na voz), mantendo as demais sensações negativas que havia referido anteriormente. Este resultado vai parcialmente ao encontro de muitos estudos com ETVSO, no quais os pacientes referem melhora na autopercepção vocal $4,5,7,10,17,28$.

Em estudo com uma sequência de quatro exercícios de fonação em tubos, realizada com 24 sujeitos disfônicos, fez-se uso de um protocolo de autoavaliação das sensações vocais e verificou-se predomínio de sensações positivas, tais como sensação de garganta aberta, maior estabilidade vocal e músculos mais relaxados ${ }^{17}$. Outras sensações referidas em estudos com ETVSO abrangem voz mais limpa, clara, firme, segura, aberta, equilibrada, solta, aveludada, suave e saindo com mais naturalidade; voz mais fácil e melhor ${ }^{28}$; voz mais solta para falar, melhora da projeção da voz e músculos mais soltos.

Analisando-se os resultados dos protocolos de qualidade de vida e voz, verifica-se que a paciente referiu melhora em relação à sua qualidade de vida na maioria dos aspectos dos protocolos, concordando com as melhoras laríngeas, vocais acústicas e perceptivo auditivas, após três semanas de terapia. No entanto, o domínio "orgânico" do IDV, a "autopercepção da voz" do PPAV e a "limitação" da ESV, pioraram. O domínio orgânico do IDV foca em aspectos específicos da produção vocal, no quanto a voz do paciente é imprevisível, no desconforto gerado e no esforço necessário para transmitir a mensagem de maneira clara e inteligível, sendo semelhante aos itens do aspecto limitação da ESV. Provavelmente, esses aspectos e a autopercepção da voz eram os que mais interferiam na qualidade de vida da paciente e, devido às limitações que o seu diagnóstico laríngeo impunha, possivelmente os resultados da terapia não tenham suprido as expectativas da paciente em relação a tais aspectos. Assim, confirma-se o que é apontado pela literatura sobre o fato de que, apesar de existir correlação significativa entre o grau de disfonia e a baixa qualidade de vida em voz, os distúrbios vocais influenciam a qualidade de vida de cada sujeito de forma particular ${ }^{13,29,30}$. Os protocolos de qualidade de vida e voz se complementam e isso justifica a importância da sua aplicação conjunta, a fim de se obter dados mais completos em relação às limitações que a disfonia ocasiona para o paciente. $\mathrm{Na}$ EHAD os escores sugestivos de depressão se encontravam em grau discreto e passaram para a faixa de normalidade e os escores de ansiedade estavam acima do ponto de corte para casos clínicos, diminuindo após a terapia ${ }^{15,31}$. Ressalta-se que a paciente já se encontrava em atendimento especializado e o manteve ao longo da fonoterapia, de forma que não é possível estabelecer se essa melhora nos escores sugestivos de depressão e ansiedade ocorreu devido ao "sentir-se cuidada" com a TBI com o FK ${ }^{31}$, devido ao tratamento psiquiátrico, ou a ambos.

Nos demais protocolos, todos os aspectos mostraram valores ainda referentes à disfonia antes e após a terapia, apesar de a maioria ter apresentado melhora após três semanas de tratamento. Com isso, pode-se verificar o quanto a presença da AL da paciente, apesar de ter melhorado no exame ORL, ainda interferiu na qualidade de vida pós-terapia. Esse aspecto pode variar entre os indivíduos, ou seja, a melhora obtida no presente estudo com outros sujeitos poderia ter evidenciado resultados de autoavaliação melhores ou piores, dependendo da importância da comunicação oral para cada um, assim como a demanda que cada profissão exige $13,29,30$. Nesse sentido, a aplicação dos protocolos fornece informações importantes que diferem em cada amostra estudada e são fundamentais para compreender as limitações impostas pela disfonia para cada sujeito individualmente ${ }^{13,29,30}$.

$\mathrm{Na}$ avaliação vocal perceptivo auditiva, verificou-se melhora de todos os aspectos avaliados, menos da astenia, que apresentou menos de $4 \%$ tanto na avaliação como na reavaliação (Tabela 5). Resultado concordante com investigação com professoras disfônicas que mostrou melhora da soprosidade e da instabilidade no grupo que não apresentava AL e melhora da instabilidade no grupo com AL, após única sessão com FK ${ }^{7}$. Além disso, com sujeitos sem disfonia, pesquisa eviden- 
ciou diminuição percentual da instabilidade vocal imediatamente após uma sessão com FK ${ }^{4}$. Outro estudo, em que os pacientes realizaram apenas um minuto de treino com o $\mathrm{FK}$, não evidenciou modificações na avaliação perceptivo auditiva ${ }^{10}$. Utilizando outras técnicas vocais, outros trabalhos mostraram melhoras sobre os aspectos perceptivo auditivos em pacientes com afecções estruturais na laringe usando a modalidade de TBI ${ }^{8}$, assim como utilizando outras modalidades de terapia ${ }^{32}$.

É importante salientar que a rouquidão e a tensão foram os dois parâmetros com maior diferença entre pré e pós-fonoterapia e pode-se fazer uma relação entre a diminuição da rouquidão, a diminuição das medidas acústicas de ruído e de jitter (Tabela 3) ${ }^{18}$ e entre a diminuição da tensão com o aumento das medidas de shimmer, VTI, DUV e NUV que sugerem menor força de fechamento glótico ou menor contato entre as pregas vocais durante a fonação ${ }^{18}$.

Quanto aos aspectos da avaliação videolaringoestroboscópica, houve melhora da amplitude de vibração da mucosa das pregas vocais, que se relaciona à diminuição dos parâmetros de rouquidão, soprosidade e aspereza obtidos, bem como das medidas acústicas de ruído e de jitter, e redução da constrição medial do vestíbulo laríngeo (Tabela 6), que se relaciona com a diminuição da tensão e o aumento das medidas de shimmer, VTI, DUV e NUV; bem como das várias melhoras em diversos domínios dos protocolos de autoavaliação vocal.

É importante salientar que, na reavaliação ORL, o médico decidiu encaminhar a paciente para exame de eletroneuromiografia laríngea, devido a suspeita de paralisia bilateral do nervo laríngeo superior, secundária à cirurgia de tireoide. Esta suspeita poderia explicar os valores de f0 rebaixados encontrados tanto na avaliação quanto na reavaliação (Tabela 2), uma vez que sem a atuação dos músculos cricotireoideos, inervados pelo laríngeo superior, a f0 torna-se diminuída ${ }^{18,19,24}$.

Apesar das melhoras evidentes após a TBI com FK, é importante reiterar que a paciente apresentava disfonia orgânica com alteração estrutural irreversível da fonte glótica e que sua disfonia permaneceu presente, embora diminuída/minimizada após a terapia escolhida e a técnica utilizada. Desta forma, não era esperado que todos os valores de suas avaliações objetivas e subjetivas passassem para a faixa de normalidade, mas que se adequassem o suficiente para que a paciente pudesse sentir-se melhor tanto social como profissionalmente, sendo o que ocorreu, conforme suas autoavaliações.

\section{Conclusão}

Após programa de TBI com a técnica FK em paciente com disfonia orgânica por fibrose em prega vocal direita, fenda glótica do tipo irregular, com assimetria de fase e amplitude, além de edema da subglote e retração da porção anterior da prega vestibular direita decorrente de intubação orotraqueal, verificaram-se modificações vocais positivas em nível glótico, respiratório e ressonantal, bem como no exame videolaringoestroboscópico e na autoavaliação vocal, evidenciando que essa foi uma modalidade de tratamento efetiva para o caso.

\section{Referências bibliográficas}

1 Martins RHG, Braz JRC, Dias NH, Castilho EC, Braz LG, Navarro LHC. Rouquidão após intubação traqueal. Rev Bras Anestesiol. 2006; 56(2): 189-99.

2 Mota LAA, Cavalho GB, Brito VA. Complicações laríngeas por intubação orotraqueal: Revisão da literatura. Int Arch Otorhinolaryngol. 2012; 16(2): 236-45.

3 Patel R, Bless DM, Thibeault SL. Boot Camp: A Novel intensive approach to voice therapy. J Voice. 2011; 25(5): 562-69.

4 Christmann MK. Modificações vocais produzidas pelo Finger Kazoo. [Dissertação]. Santa Maria (RS): Universidade Federal de Santa Maria; 2012.

5 Cielo CA, Christmann MK. Finger kazoo: modificações vocais acústicas espectrográficas e autoavaliação vocal. Rev CEFAC. 2014; 16(4):1239-54.

6 Cielo CA, Christmann MK, Frigo LF. Evidência científica da técnica finger kazoo. In: Terapia fonoaudiológica baseada em evidências. Vol I. Barueri: Org Pró-Fono. 2013; cap. 19, p.357-70.

7 Bastilha GR. Efeitos vocais imediatos da técnica finger kazoo em professoras disfônicas com e sem afecções laríngeas. [Dissertação]. Santa Maria (RS):

Universidade Federal de Santa Maria; 2015.

$8 \mathrm{Fu} \mathrm{S}$, Theodoros DG, Ward EC. Intensive versus traditional voice therapy for vocal nodules: perceptual, physiological, acoustic and aerodynamic changes. J Voice. 2015; 29(2): 31-44.a.

9 Fu S, Theodoros DG, Ward EC. Delivery of intensive voice therapy for vocal fold nodules via telepractice: A pilot feasibility and efficacy study. J Voice. 2015; 29(6): 696-706.b.

10 Sampaio M, Oliveira G, Behlau M. Investigação de efeitos imediatos de dois exercícios de trato vocal semiocluído. PróFono. 2008: 20(4): 261-6.

11 Ricarte A, Oliveira G, Behlau M. Validação do protocolo Perfil de Participação e Atividades Vocais no Brasil. CoDAS. 2013; 25(3): 242-9. 
12 Zambon F, Moreti F, Vargas ACV, Behlau M. Eficiência e valores de corte do Perfil de Participação e Atividades Vocais. CoDAS. 2015; 27(6): 598-603.

13 Madazio G, Moreti F, Yamasaki R. Protocolos de autoavaliação do impacto da disfonia. In: Tratado de especialidades em fonoaudiologia. São Paulo: Guanabara Koogan. 2014; cap. 16, p.113-26.

14 Moreti F, Zambon F, Oliveira G, Behlau M. Cross-cultural adaptation, validation, and cutoff values of the Brazilian version of the Voice Symptom Scale-VoiSS. J Voice. 2014; 28(4): 458-68.

15 Mathieson L, Hirani SP, Epstein R, Baken RJ, Wood G, Rubin JS. Laryngeal manual therapy: a preliminary study to examine its treatment effects in the management of muscle tension dysphonia. J Voice. 2009; 23 (3): 353-66.

16 Marcolino, JAM, Mathias LAST, Piccinini FL, Guaratini AA, Suzuki FM, Alli LAC. Escala Hospitalar de Ansiedade e Depressão: Estudo da validade de critério e da confiabilidade com pacientes no pré-operatório. Rev Bras Anestesiol. 2007; 57(1): 52-62.

17 Guzmán M, Angulo M, Muñuz D, MayerhoffR. Effect on long-term average spectrum of pop singers' vocal warm-up with vocal function exercises. International J Speech Language Pathol. 2012; 15(2): 127-35.

18 Behlau M. O livro do especialista. Rio de Janeiro: Revinter, 2008,348 p.

19 Colton RH, Casper JK, Leonard D. Compreendendo os problemas de voz: uma perspectiva fisiológica ao diagnóstico e ao tratamento. Rio de Janeiro: Revinter, 2010. 445p.

20 Côrtes MG, Gama ACC. Análise visual de parâmetros espectrográficos pré e pós-fonoterapia para disfonias. Rev Soc Bras Fonoaudiol. 2010; 15(2): 243-9.

21 Pedrosa V, Pontes A, Pontes P, Behlau M, Peccin SM. The effectiveness of the comprehensive voice rehabilitation program compared with the vocal function exercises method in behavioral dysphonia: A randomized clinical trial. J Voice. 2016; 30(3): 377.e11-9.

22 Pinho SMR, Pontes P. Escala de avaliação perceptiva da fonte glótica: RASAT. Vox Brazilis. 2002; 3(1): 11-3.

23 Verdolini-Marston K, Sandage M, Titze IR. Effect of hydration treatments on laryngeal nodules and polyps and related voice measures. J Voice. 1994; 8(1): 30-47.

24 Saxon KG, Schneider CM. Vocal exercise physiology. California: Singular Publishing Group, 1995.

25 Cielo CA, Lima JPM, Gonçalves BFT, Christmann MK. Relações entre /s/e /z/ e entre /e/ e /e/ não vozeado ou áfono. Rev CEFAC. 2013; 15(5): 1308-15.

26 Mendonça RA, Sampaio TMM, Provenzano L. Medida do tempo máximo de fonação de professoras do município de Niterói/RJ. Rev CEFAC. 2012; 14 (6): 1204-8.

27 Cielo CA, Gonçalves BFT, Lima JPM, Christmann MK. Tempo máximo de fonação /a/, tempo máximo de fonação previsto e tipo respiratório de mulheres adultas sem afecções laríngeas. Rev CEFAC. 2015; 17(2): 358-63.

28 Maia MEO, Maia MO, Gama ACC, Behlau M. Efeitos imediatos do exercício vocal sopro e som agudo. J Soc Bras Fonoaudiol. 2012; 24(1): 1-6.
29 Tutya AS, Zambon F, Oliveira G, Behlau M. Comparação dos escores dos protocolos QVV, IDV e PPAV em professores. Rev Soc Bras Fonoaudiol. 2011; 16(3): 273-81.

30 Cielo CA, Ribeiro VV. Autoavaliação vocal de professores de Santa Maria/RS. Rev CEFAC. 2015; 17(4): 1152-60.

31 Martinez CC, Cassol M. Measurement of voice quality, anxiety and depression symptoms after speech therapy. J Voice. 2015; 29(4): 446-9.

32 Schindler A, Mozzanica F, Maruzzi P, Atac M, Cristofaro $\mathrm{V}$, Ottaviani F. Multidimensional assessment of vocal changes in benign vocal fold lesions after voice therapy. Auris Nasus Larynx. 2013; 40(1): 291-7. 


\section{ANEXO 1}

\section{Questionário das sensações subjetivas em relação à voz}

Nome:

Data:

\section{SENSAÇÕES EM RELAÇÃO À VOZ:}

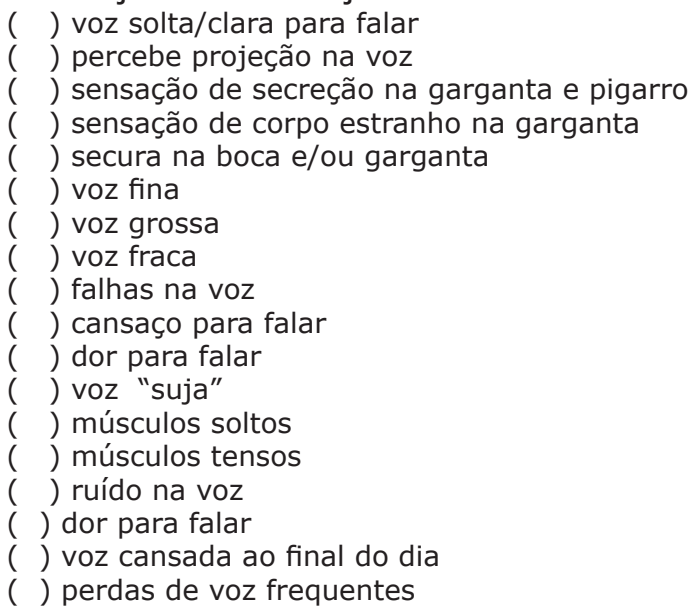

OUTRAS SENSAÇÕES: 